\title{
Qualifizierter Entzug
}

\section{Die stationäre Entzugsbehandlung von Alkoholabhängigen und ihre ambulante Fortführung}

\section{B. Croissant, K. Mann}

Zentralinstitut für Seelische Gesundheit, Klinik für Abhängiges Verhalten und Suchtmedizin,Avrannh $\mathrm{m}$ (Direktor: Prof. Dr. K. Mann)

Primäres Ziel der traditionellen Entgiftungsbehandlung ist es, mithilfe einer adäquaten medikamentösen Behandlung die Entzugserscheinungen zu beherrschen und das Überleben des Patienten sicherzustellen. Spezifische Maßnahmen gegen die Grunderkrankung der Abhängigkeit werden bisher nur in wenigen Fällen durchgeführt. Die Nachuntersuchungszahlen dieser traditionellen Entgiftung sind außerordentlich schlecht. Suchtmedizinisch geschulte Liaison-Dienste könnten aber bei der stationären Entgiftung in Allgemeinkrankenhäusern wesentliche Verbesserungen des bestehenden Versorgungssystems erreichen. Ziel dieser Maßnahmen ist die konsequente Erarbeitung einer hinreichenden Krankheitseinsicht, sodass die Patienten mit mehr Erfolg in weiterführende Behandlungen vermittelt werden könnten. Mit der Einführung des DRG-Systems („diagnosis related groups“) wird die Fallkostenkonstellation der „qualifizierten Entzugsbehandlung“ jedoch nicht abgebildet werden können. Soll diese jedoch - mit einem entsprechenden multiprofessionellen Team - in der Inneren Abteilung durchgeführt werden, deckt die Situation nach DRG bislang keinesfalls die Kosten. Somatische Abteilungen sollten durch eine geeignete Finanzierung in die Lage versetzt werden, dieses anspruchsvolle, von Ärzten, Psychologen und Sozialarbeitern gemeinsam durchgeführte und im Gesamtverlauf kostensparende Angebot der „qualifizierten Entgiftung“ hinsichtlich Behandlungsdauer und Personalausstattung angemessen zu realisieren.

Ü bergeordnetes Ziel jeder ärztliche Therapie alkoholkranker Menschen muss heute sein, zum einen die Abstinenzmotivation und zum anderen die Krankheitseinsicht in die Grunderkrankung (Abhängigkeit) zu fördern. Von wesentlicher Bedeutung in diesem prozesshaften therapeutischen Geschehen ist die Entgiftungsbehandlung. Denn es gelingt nur einer begrenzten Zahl von Alkoholkranken, unmittelbar nach der Entgiftung eine mehrmonatige stationäre Entwöhnungsbehandlung anzutreten. Weitaus mehr Pati- enten unterziehen sich im Laufe ihrer Suchterkrankung einer oder wiederholten Entgiftungsbehandlungen, zu denen sie in vielen Fällen von ihren betreuenden Ärzten zunächst leichter zu motivieren sind. Da in Allgemeinkrankenhäusern Entgiftungsbehandlungen nur wenige Tage dauern, bieten sie allein schon aufgrund dieser zeitlichen Limitation nur in Ausnahmefällen eine ausreichende Hilfestellung, um anschließend ein abstinentes Leben zu führen.

Eine Entgiftung muss grundsätzlich eine eingehende somatische und psychiatrische Diagnostik und die Behandlung von Entzugssymptomen und Folgeerkrankungen enthalten. Zur „qualifizierten Entzugsbehandlung“" (Bundesministerium für Jugend, Familie, Frauen und Gesundheit, Expertenkommission 1988) wird sie jedoch erst, wenn motivationsfördernde Interventionen in multidisziplinären Teams (Ärzte, Psychologen, Sozialarbeiter) einbezogen werden, welche darauf abzielen, die weitere Behandlungsbereitschaft und -fähigkeit der $\mathrm{Pa}$ tienten zu fördern.

\section{Stationäre Entgiftungen}

Primäres Ziel der traditionellen Entgiftungsbehandlung ist es, mithilfe einer adäquaten medikamentösen Behandlung der vegetativen Entzugserscheinungen oder eines Delirium tremens das Überleben des Patienten sicherzustellen $(1,7)$. Spezifische Maßnahmen gegen die Grunderkrankung der Abhängigkeit erfolgen bisher jedoch nur in weniger als 10\% der Fälle (5). Die Nachuntersuchungszahlen dieser traditionellen Entgiftung sind mit rund 40\% Verstorbenen und nur 5\% Abstinenten nach durchschnittlich acht Jahren außerordentlich schlecht (12). Entsprechend häufig müssen die Patien- 
ten zu weiteren Entgiftungen in die stationäre Behandlung zurückkehren (3). Somit wird das Überleben akut zwar gesichert, die Einsicht in die Grundkrankheit und die Motivation zur Veränderung des Verhaltens mit dem endgültigen Ziel der Abstinenz werden jedoch nicht systematisch gefördert und kaum erreicht.

Darüber hinaus führen wiederholte Entzüge zu immer gravierenderen Schäden, zum Beispiel durch exzitotoxische Schädigungen von Neuronen (4). Sprechen die in das Entgiftungsgeschehen involvierten Neurotransmittersysteme von mal zu mal stärker auf den Entzug an und ist dies mit einer Kaskade weiterer Folgen - wie beispielsweise Krampfanfälle - verbunden, spricht man von einem Kindlingphänomen (9). Ob jemand drei oder 13 Entgiftungen mitmacht, ist also nicht ohne Bedeutung.

Als deletär erwies sich die alte Vorstellung, dass besonders schwere Entzüge eine besonders gute Motivation für weiterführende Behandlungen erzielen würden. Auch der frühere therapeutische Grundsatz, wonach die Patienten erst an einem Tiefpunkt angekommen sein müssen, um entsprechende Schritte einzuleiten, ist aus heutiger Sicht obsolet. Leider führt der administrative Aufwand infolge der Trennung der Zuständigkeiten zwischen Krankenkassen und Rentenversicherern immer noch zu Wartezeiten und zu einer unbefriedigenden Inanspruchnahme der Rehabilitationsbehandlung, womit erneute Entgiftungen mit allen geschilderten Konsequenzen nötig werden können.
Bei der stationären Entgiftung in Allgemeinkrankenhäusern können so genannte Liaison-Dienste das bestehende Versorgungssystem wesentlich verbessern. Dabei übernimmt eine suchtmedizinisch geschulte Fachkraft die Weiterbildung und Supervision der Ärzte sowie des Pflegepersonals. Von Fall zu Fall können auch direkte Patientenkontakte wahrgenommen werden. So könnte eine zusätzliche Fachkraft im Rahmen eines Alkohol-Liaison-Dienstes für etwa 320 Betten zuständig sein (10). Mit diesen Maßnahmen kann die Rate der Vermittlung in weiterführende Therapieangebote nahezu verdoppelt werden (9). Darüber hinaus tragen sowohl eine bessere Fortbildung (Zusatzqualifikation „suchtmedizinische Grundversorgung“, etwa 50 Stunden) als auch der Einsatz neuer rückfallprophylaktischer Medikamente dazu bei, die ärztlichen Möglichkeiten innerhalb des Therapienetzes deutlich zu erweitern.

\section{Qualifizierte}

Entzugsbehandlung

Zudem könnte auch eine bessere therapeutische Nutzung der Phase der körperlichen Entgiftung die Versorgung von Alkoholkranken verbessern. Anstatt die Entgiftung passiv zu erleiden, könnten wir diese Phase für den Abhängigen aktiv gestalten. Ansätze hierzu haben schon 1988 die Empfehlungen der Expertenkommission angedeutet. Unter dem programmatischen Titel „Keine Entgiftung ohne psychotherapeutische Begleitung“ stellten Mann und Stetter eine Konzeptualisierung dieser Ideen vor (7).
Wesentliche Merkmale der erweiterten Entgiftung, für die sich der Begriff „qualifizierte Entzugsbehandlung“ durchgesetzt hat, sind neben einer differenzierten, somatisch gut fundierten Diagnostik sowie der Behandlung der Entzugssymptome, der körperlichen Begleiterkrankungen und der Folgeerkrankungen vor allem das Fehlen abwehrender Aufnahmeprozeduren, motivationsprüfender Schwellen oder abwertender Konfrontationen.

Nach dieser Definition ist die körperliche Entzugssituation eine Chance, durch gezielte psychotherapeutische Beeinflussung Motivationsarbeit zu leisten. Ziel dieser Maßnahmen ist die konsequente Erarbeitung einer hinreichenden Krankheitseinsicht, die - über verschiedene Motivationsstrategien verstärkt - letztendlich zu der Bereitschaft des Patienten führt, eine weiterführende Behandlung anzutreten. Ein derartiger Prozess ist langwierig, sodass die Behandlungsdauer mit drei bis sechs Wochen anzusetzen ist. Dies trägt außerdem dazu bei, das protrahierte Entzugssyndrom mit seiner erhöhten Rückfall- und Suizidgefährdung besser beherrschen zu können.

Günstige Ergebnisse nach qualifiziertem Entzug präsentierten auch Forscher aus Jena, Lübeck, Mannheim und Würzburg (Tab. 1). Im Sinne von sekundärpräventiven Maßnahmen leisteten sie damit einen nachhaltigen Beitrag zu einer früheren Diagnostik und Intervention. Ihre Resultate ließen sich nicht nur anhand von Erfolgszahlen bezüglich des Trinkverhaltens belegen, sondern auch

\section{Tab. 2 Ergebnisse nach stationärer Entwöhnungsbehandlung}

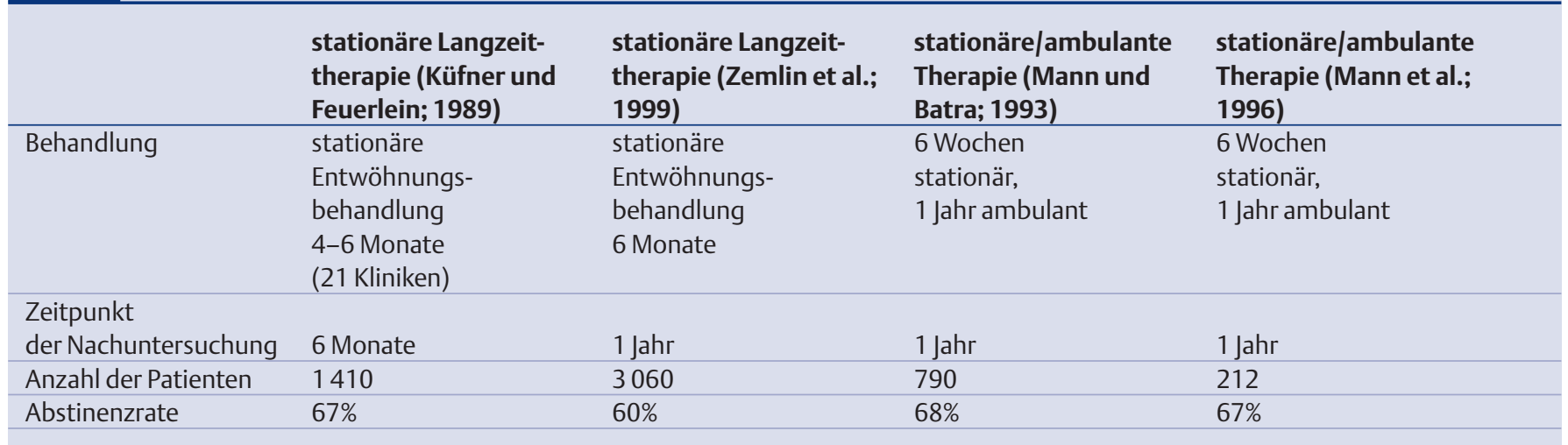


durch eine Senkung der Kosten, da in der Folgezeit signifikant weniger Krankenbehandlungen in Anspruch genommen wurden (2).

\section{Ziele der qualifizierten Entzugsbehandlung}

Neben dem primären Ziel der körperlichen Entgiftung bei akut intoxikierten Patienten geht es bei der qualifizierten Entzugsbehandlung vor allem darum, den Patienten zu akzeptieren, sein Selbstwertgefühl zu stabilisieren und eine Vertrauen fördernde Atmosphäre zu schaffen. Vor diesem Hintergrund kann die Krankheits- und Problemeinsicht sowie das Gesundheitsbewusstsein gefördert werden. Erst bei einer tragfähigen therapeutischen Beziehung gewinnen konfrontative Elemente an Bedeutung. Der Leidensdruck des Patienten, sein Hilfewunsch, seine Erfolgserwartungen und die von ihm erwarteten Kosten einer Entwöhnungstherapie werden evaluiert und die zugrunde liegenden Kognitionen und Affekte mit dem Ziel bearbeitet, die Bereitschaft zum Antritt einer ambulanten oder stationären Entwöhnungsbehandlung zu erhöhen.

Die Eigenmotivation zur Abstinenz ist - im Gegensatz zu Entwöhnungsbehandlungen - keine Eingangsvoraussetzung für eine qualifizierte Entzugstherapie. Vielmehr besteht eines der Therapieziele eben gerade darin, die Abstinenz- und die Veränderungsbereitschaft $\mathrm{zu}$ fördern. Kontrollierte Studien belegen die Effekte derartiger psychotherapeutischer Interventionen, die bereits im ambulanten Kontakt und auch in der Entgiftungsphase beginnen sollten (11).

\section{Psychotherapeutische Interventionen}

Jede Therapie sollte soweit als möglich auf die individuelle Situation und Person des jeweiligen Patienten abgestimmt werden. Dennoch lassen sich einige wesentliche Merkmale beschreiben, welche die therapeutische Arbeit in der Entzugsphase kennzeichnen (6):

- Der persönliche therapeutische Kontakt sollte so früh wie möglich hergestellt werden, damit die Krisensituation mit ihren spezifischen Möglichkeiten genutzt werden kann.

- Alle psychotherapeutischen Interventionen sollten auf die Abhängigkeitserkrankung fokussieren.

- Überschaubaren Zielen ist der Vorzug gegenüber groß angelegten „Veränderungsvorstellungen“ zu geben.

- Zwischen Patient und Therapeut und auch in therapeutischen Gruppen sollten erreichbare Schritte auf diese Nahziele hin thematisiert werden. Für die Therapeuten geht es darum, aktive Hilfestellungen zur Bewältigung der unmittelbar anliegenden Probleme zu leisten.

- Sowohl der Patient als auch der Therapeut muss akzeptieren, dass in dieser Therapiephase (bei der zumeist auf einige Tage bis wenige Wochen beschränkten Zeit), eine „zufriedene Abstinenz" als übergeordnetes Ziel zwar ins Auge gefasst werden kann. In aller Regel sind jedoch für die schwer abhängigen Patienten hierzu wesentliche Umstrukturierungen notwendig, die sich als ein monate-, oft sogar jahrelang andauernder Prozess gestalten, der weitere therapeutische Hilfe nötig macht.

- Im Sinne der „Motivationstherapie" bietet es sich daher an, die Förderung der Bereitschaft, weitere Hilfe anzunehmen, zum zentralen Therapieziel zu machen. Besonders bewährt haben sich dabei klare Aussagen in dem Sinne, dass eine Besserung der Lebenssituation durch eine weiterführende alkoholismusspezifische Behandlung zu erwarten ist.

- Darüber hinaus kann die Motivationstherapie darin bestehen, Informationen über die Krankheit mit der persönlichen Betroffenheit des Patienten und konkrete pathologische Befunde - aber auch deren Rückbildung unter Abstinenzbedingungen - mit dynamischen Prozessen zu verbinden, die Krankheitseinsicht und Motivationsförderung zum Gegenstand haben.

- Konkret und aktiv helfen kann man den Patienten darüber hinaus, indem man diesen Infor-

\section{Tab. 1 Ergebnisse nach qualifiziertem Entzug}

\begin{tabular}{|c|c|c|c|c|c|}
\hline & $\begin{array}{l}\text { Olbrich } \\
(2001)\end{array}$ & $\begin{array}{l}\text { Stetter und } \\
\text { Mann (1997) }\end{array}$ & $\begin{array}{l}\text { Veltrup } \\
(1995)\end{array}$ & $\begin{array}{l}\text { Böning } \\
\text { et al. (2001) }\end{array}$ & $\begin{array}{l}\text { Bauer und } \\
\text { Hasenöhrl (2000) }\end{array}$ \\
\hline Behandlung & $\begin{array}{l}3 \text { Wochen } \\
\text { allgemeinpsy- } \\
\text { chiatrische Station }\end{array}$ & $\begin{array}{l}3 \text { Wochen } \\
\text { Suchtstation }\end{array}$ & $\begin{array}{l}6 \text { Wochen } \\
\text { Suchtstation }\end{array}$ & $\begin{array}{l}6 \text { Wochen } \\
\text { Suchtstation }\end{array}$ & $\begin{array}{l}\text { 4-8 Wochen } \\
\text { Suchtstation }\end{array}$ \\
\hline Zeitpunkt der Nachuntersuchung & 6 Monate & 8 Monate & 8 Monate & 12 Monate & 28 Monate \\
\hline Anzahl der Patienten & 102 & 529 & 196 & 151 & 92 \\
\hline Durchführung & persönlich & telefonisch & persönlich & persönlich & postalisch \\
\hline $\begin{array}{l}\text { Abstinenzrate der } \\
\text { nachuntersuchten Patienten }\end{array}$ & $58 \%$ & $52 \%$ & $58 \%$ & $45 \%$ & $51 \%$ \\
\hline $\begin{array}{l}\text { Abstinenzrate bezogen } \\
\text { auf die Ausgangsstichprobe }\end{array}$ & $48 \%$ & $46 \%$ & $38 \%$ & $36 \%$ & $32 \%$ \\
\hline $\begin{array}{l}\text { Vermittlung in weiterführende } \\
\text { Behandlung }\end{array}$ & & $46 \%$ & & & \\
\hline
\end{tabular}


mationen über weitere Behandlungsmöglichkeiten, insbesondere über die ambulante oder die stationäre Entwöhnung gibt und mit ihnen die nächsten Schritte vereinbart, die zur Einleitung derartiger Maßnahmen führen.

- Die Kontaktaufnahme zu Betroffenen, die „es geschafft haben“, kann den Patienten helfen, eine Perspektive $\mathrm{zu}$ gewinnen und Ratschläge anzunehmen, die aus dem Munde von Therapeuten möglicherweise ihre Wirkung verfehlt hätten.

Bei der qualifizierten Entzugsbehandlung handelt es sich um eine eigenständige suchtspezifische Form einer Krankenhausbehandlung für Abhängige. Dafür müssen mehr Ressourcen und längere Behandlungszeiten vorgesehen werden als für die bisher völlig unzureichenden „Entgiftungen“, von denen die Kalkulationen gegenwärtig ausgehen.

\section{- Bedeutung der DRGs}

In Deutschland ist das bisherige Entgeltsystem ein Mischsystem aus tagesgleichen Pflegesätzen, Fallpauschalen und Sonderentgelten. Kranken- und Rentenversicherung teilen die Kosten für die Behandlung auf der Basis der „Vereinbarung Abhängigkeitserkrankungen“ vom 1. Juli 2001 unter sich auf. Dabei übernimmt die Krankenversicherung im Normalfall die „Entgiftungsphase“ und die Rentenversicherung die „Entwöhnungsphase“. Letztere ist eine Behandlung der „medizinischen Rehabilitation“, die auf Antrag erfolgt, und deren Ziel - zumindest von Seiten der Rentenversicherung die „Verbesserung bzw. Wiederherstellung der Erwerbsfähigkeit“ ist.

Momentan bestehen insbesondere bei der Kostenübernahme durch die gesetzliche Krankenversicherung Schwierigkeiten, was die Dauer des qualifizierten Entzugs im Sinne einer medizinischen Akutbehandlung betrifft. In einigen Bundesländern (z.B. Berlin und Bayern) wird sehr rigide verfahren, obwohl der rechtliche Anspruch des Patienten begründet ist (9). In Nordrhein-
Westfalen dagegen gibt es eine einvernehmlich zwischen Krankenund Rentenversicherern sowie den Krankenhausträgern vereinbarte „Drei-Wochen-Regelung“.

Die Behandlungsdauer im Allgemeinkrankenhaus steht ebenso unter Druck wie die „qualifizierte Entzugsbehandlung“ zu Lasten der gesetzlichen Krankenversicherung (GKV). Vor diesem Hintergrund ist die Diskussion über die Einführung der „diagnosis related groups“ (DRGs) in Krankenhäuser der Allgemeinversorgung zu sehen. Nach dem Gesundheitsreform-Gesetz 2000 wird in Deutschland ein pauschalierendes Entgeltsystem eingeführt werden. Der Erlös (Preis) für die Behandlung eines Patienten ergibt sich dabei im Wesentlichen aus der zur Krankenhausbehandlung führenden Hauptdiagnose, komplizierenden Begleiterkrankungen und der medizinischen Bezugsleistung. In Deutschland wird zu diesem Zweck das australische DRG-System adaptiert.

Dabei werden noch einmal alle bisher auch schon kontrovers diskutierten Fragen von Zuständigkeit, Planung und Entwicklung von regionaler Versorgung angesprochen. Die am häufigsten gestellten Fragen dabei sind:

- Reichen die Fallpauschalen in den Allgemeinkrankenhäusern für eine umfängliche Behandlung allein der somatischen Störungen aus?

- Sind internistische Stationen in der Lage, eine Entzugsbehandlung durchzuführen, die den derzeitigen Standards - das heißt der Einbeziehung zumindest von Motivationsanteilen Rechnung trägt?

- Wird es nach einer Anfangsbehandlung im Allgemeinkrankenhaus zu einer vermehrten Verlegung in psychiatrische Krankenhäuser kommen?

- Wird es möglich sein, psychiatrische Kompetenz im Allgemeinkrankenhaus in die Behandlung einzubinden?

- Wird der Druck auf die Patienten, eine medizinische Rehabilitation zu beantragen, noch zunehmen?
- Wird bei knappen finanziellen Vorgaben die Möglichkeit der regionalen Zusammenarbeit und eines fachlichen Austausches noch gegeben sein?

Die Fallkostenkonstellation der „qualifizierten Entzugsbehandlung“ bildet das DRG-System bisher nicht ab. Dies ist für qualifizierte Entzugsbehandlungen, die in der Psychiatrie stattfinden, auch nicht nötig, denn bislang fällt die Psychiatrie gemäß $\S 17 \mathrm{~b}$ KHG nicht unter die DRGs. Soll die qualifizierte Entzugsbehandlung bei Alkoholabhängigkeit (ICD 10 F10.2) jedoch in der Inneren Abteilung durchgeführt werden, ist die Kostengewichtung keinesfalls kostendeckend. Die Kalkulationen für die DRG V60Z (Alkoholintoxikation und -entzug) legt eine mittlere Verweildauer von 2,6 Tagen und für die V62A (Störungen durch Alkoholmissbrauch und Alkoholabhängigkeit) von 6,8 Tagen zugrunde. Mit solchen mittleren Verweildauern lässt sich der dem Stand der Wissenschaft entsprechende qualifizierte Entzug nicht gewährleisten.

Somatische Abteilungen sollten durch eine geeignete Finanzierung in die Lage versetzt werden, dieses anspruchsvolle, bewährte und im Gesamtverlauf kostensparende Angebot der qualifizierten Entgiftung (2) hinsichtlich Behandlungsdauer und Personalausstattung angemessen zu realisieren. Aus diesen Gründen wurde die Einrichtung einer neuen DRG „Qualifizierter Entzug bei Störungen durch Alkohol und/ oder Medikamentenabhängigkeit“ gesteuert über einen neuen Operationsschlüssel (OPS-301 nach § 301 Sozialgesetzbuch V) „qualifizierter Entzug“ - beantragt. Der Operationsschlüssel wird in der stationären Versorgung eingesetzt zur Verschlüsselung der medizinischen Prozeduren für die Abrechnung von Krankenhausleistungen nach den Sonderentgelten und Fallpauschalen sowie nach dem System der DRG.

\section{Ambulante Nachbetreuung}

Im Anschluss an eine (teil-)stationäre Entzugs- oder Entwöhnungstherapie sollte unbedingt eine ambulante Nachbetreuung angebo- 
ten werden. Denn diese unterstützt die Patienten bei der Wiedereingliederung in ihr Alltagsleben und bietet eine schnelle und für die Patienten unkompliziert zu erreichende Interventionsmöglichkeit während akuter psychischer Krisen mit hoher Rückfallgefährdung. Der Schwerpunkt einer ambulanten Nachbetreuung ist die Beibehaltung der Abstinenz und die Stabilisierung der in der (teil-)stationären Phase initiierten Verhaltensänderung.

Eine besondere Rolle sollte hier der Zusammenarbeit psychosozialer Beratungsstellen (z.B. Caritas, Diakonie) mit niedergelassenen Ärzten zukommen. Dies setzt allerdings eine wesentlich bessere Aus-, Weiter- und Fortbildung in Suchtmedizin („Fachkunde Suchtmedizin“, s.o.) sowie eine angemessene Honorierung voraus. Neben der regelmäßigen ambulanten Betreuung der leichter Abhängigen und weniger Geschädigten durch Haus- und Fachärzte hat sich die regelmäßige Teilnahme an Selbsthilfegruppen bewährt (zum Beispiel Anonyme Alkoholiker, Blaukreuzler, Guttempler). Von besonderer Bedeutung ist die medikamentöse Therapie der Rückfallprophylaxe, wie sie in diesem Heft ebenfalls beschrieben ist.

\section{Qualified Withdrawal Treatment - Inpatient Detoxification for Alcohol Addicted Patients and its Ambulant Continuation}

The primary aim of the traditional detoxification treatment is to guarantee the survival of the patient with the help of adequate medication. Up to now specific procedures against the underlying dependence are adopted in a few cases only. The reevaluations of this traditional detoxification show exceptionally poor results. For inpatient detoxification in general hospitals essential improvements of the existing care system can be achieved with the help of liaisonservices trained in addiction medicine. Motivational techniques are added to standard acute treatment while the aim of these interventions is the consistent development of a sufficient self insight in the disease which finally leads to the willingness of the patient to enter further abstinence-maintaining treatments. According to the health reform-law 2000 an all inclusive remuneration system is being introduced in Germany. For this purpose the Australian DRG (Diagnosis Related Groups)-system is adapted. The expenses for the treatment constellation „Qualified withdrawal treatment" are not represented in this system. If the qualified withdrawal treatment in alcohol dependence (ICD $10 \mathrm{~F} 10.2$ ) is to be carried out in general hospitals - with a multiprofessional team - the expenses are in no way covered by the DRG. Somatic departments should be enabled to realize this demanding and in the whole course cost-saving offer of the „qualified withdrawal treatment" which is provided by physicians, psychologists and social workers in common with regard to treatment duration and personnel equipment by a suitable financing.

\section{Key Words}

qualified withdrawal treatment outpatient after care - inpatient withdrawal - diagnosis related groups - lump compensation

\section{Literatur bei den Verfassern, bei der Redaktion}

Anschrift für die Verfasser
Dr. Bernhard Croissant
Zentralinstitut für Seelische Gesundheit
Klinik für Abhängiges Verhalten und
Suchtmedizin
Leitender Oberarzt der Klinik (komm.)
J5
68159 Mannheim

